Journal for ImmunoTherapy of Cancer

\title{
Phase 2 study of cemiplimab in patients with metastatic cutaneous squamous cell carcinoma: primary analysis of fixed-dosing, long-term outcome of weight-based dosing
}

Danny Rischin, ${ }^{1}$ Michael R Migden, ${ }^{2}$ Annette M Lim, ${ }^{1}$ Chrysalyne D Schmults, ${ }^{3}$ Nikhil I Khushalani, ${ }_{4}$ Brett G M Hughes, ${ }^{5}$ Dirk Schadendorf, ${ }^{6}$ Lara A Dunn, ${ }^{7}$ Leonel Hernandez-Aya, ${ }^{8}$ Anne Lynn S Chang, ${ }^{9}$ Badri Modi, ${ }^{10}$ Axel Hauschild, ${ }^{11}$ Claas Ulrich, ${ }^{12}$ Thomas Eigentler, ${ }^{13}$ Brian Stein, ${ }^{14}$ Anna C Pavlick, ${ }^{15}$ Jessica L Geiger, ${ }^{16}$ Ralf Gutzmer, ${ }^{17}$ Murad Alam, ${ }^{18}$ Emmanuel Okoye, ${ }^{19}$ Melissa Mathias, ${ }^{20}$ Vladimir Jankovic, ${ }^{20}$ Elizabeth Stankevich, ${ }^{20}$ Jocelyn Booth, ${ }^{21}$ Siyu Li, ${ }^{21}$ Israel Lowy, ${ }^{20}$ Matthew G Fury, ${ }^{20}$ Alexander Guminski ${ }^{22}$

To cite: Rischin D, Migden MR, Lim AM, et al. Phase 2 study of cemiplimab in patients with metastatic cutaneous squamous cell carcinoma: primary analysis of fixeddosing, long-term outcome of weight-based dosing. Journal for ImmunoTherapy of Cancer 2020;8:e000775. doi:10.1136/ jitc-2020-000775

\section{- Additional material is} published online only. To view please visit the journal online (http://dx.doi.org/10.1136/jitc2020-000775).

Accepted 07 May 2020

Check for updates

(C) Author(s) (or their employer(s)) 2020. Re-use permitted under CC BY-NC. No commercial re-use. See rights and permissions. Published by BMJ.

For numbered affiliations see end of article.

Correspondence to Professor Danny Rischin; danny.rischin@petermac.org

\section{ABSTRACT}

Background Cemiplimab, a high-affinity, potent human immunoglobulin G4 monoclonal antibody to programmed cell death-1 demonstrated antitumor activity in a Phase 1 advanced cutaneous squamous cell carcinoma (CSCC) expansion cohort (NCT02383212) and the pivotal Phase 2 study (NCT02760498). Here we report the primary analysis of fixed dose cemiplimab $350 \mathrm{mg}$ intravenously every 3 weeks (Q3W) (Group 3) and provide a longer-term update after the primary analysis of weight-based cemiplimab $3 \mathrm{mg} / \mathrm{kg}$ intravenously every 2 weeks (Q2W) (Group 1) among metastatic CSCC (mCSCC) patients in the pivotal study (NCT02760498).

Methods The primary objective for each group was objective response rate (ORR) per independent central review (ICR). Secondary endpoints included ORR by investigator review (INV), duration of response (DOR) per ICR and INV, and safety and tolerability.

Results For Group $3(n=56)$ and Group $1(n=59)$, median follow-up was 8.1 (range, 0.6 to 14.1) and 16.5 (range, 1.1 to 26.6 ) months, respectively. ORR per ICR was $41.1 \%$ (95\% Cl, $28.1 \%$ to $55.0 \%)$ in Group 3, $49.2 \%(95 \% \mathrm{Cl}$, $35.9 \%$ to $62.5 \%)$ in Group 1, and $45.2 \%$ (95\% Cl, $35.9 \%$ to $54.8 \%$ in both groups combined. Per ICR, KaplanMeier estimate for DOR at 8 months was $95.0 \%(95 \% \mathrm{Cl}$, $69.5 \%$ to $99.3 \%$ ) in responding patients in Group 3, and at 12 months was $88.9 \%(95 \% \mathrm{Cl}, 69.3 \%$ to $96.3 \%)$ in responding patients in Group 1. Per INV, ORR was $51.8 \%$ (95\% Cl, $38.0 \%$ to $65.3 \%)$ in Group 3, $49.2 \%(95 \% \mathrm{Cl}$, $35.9 \%$ to $62.5 \%)$ in Group 1, and $50.4 \%$ (95\% Cl, $41.0 \%$ to $59.9 \%$ ) in both groups combined. Overall, the most common adverse events regardless of attribution were fatigue $(27.0 \%)$ and diarrhea (23.5\%).

Conclusion In patients with mCSCC, cemiplimab $350 \mathrm{mg}$ intravenously Q3W produced substantial antitumor activity with durable response and an acceptable safety profile. Follow-up data of cemiplimab $3 \mathrm{mg} / \mathrm{kg}$ intravenously Q2W demonstrate ongoing durability of responses.
Trial registration number Clinicaltrials.gov, NCT02760498. Registered May 3, 2016, https:// clinicaltrials.gov/ct2/show/NCT02760498

\section{INTRODUCTION}

Cutaneous squamous cell carcinoma (CSCC) is the second most common skin cancer, and its incidence is increasing. ${ }^{12}$ Chronic sun exposure, advanced age, and immunosuppression are risk factors for CSCC. ${ }^{3} 4$ Most CSCC cases are diagnosed early, ${ }^{56}$ and patients with local disease are generally cured by surgery. ${ }^{4}$ Conversely, the prognosis is poor for patients with either locally advanced CSCC (laCSCC) not amenable to curative surgery or curative radiation or metastatic CSCC (mCSCC), collectively referred to as advanced CSCC, treated with cytotoxic chemotherapy or epidermal growth factor receptor inhibitors. ${ }^{8-10}$

Due to chronic skin damage from ultraviolet light, most CSCCs are hypermutated. ${ }^{11} 12$ Patients with high tumor mutational burden (TMB) solid tumors are more likely to derive clinical benefit from inhibition of immune checkpoints, such as programmed cell death (PD)-1. ${ }^{13} 14$ Intact immune surveillance is critical in CSCC prevention in immunocompetent individuals, as evidenced by the strong link between immunosuppression and increased CSCG risk. ${ }^{15} 16$ These considerations provided rationale for the study of PD-1 inhibition in advanced CSCC. 
Cemiplimab is a high-affinity, highly potent human immunoglobulin G4 monoclonal antibody to the PD-1 receptor. ${ }^{17}$ Cemiplimab demonstrated substantial antitumor activity in a Phase 1 advanced CSCC expansion cohorts (NCT02383212) and produced an objective response rate (ORR) per independent central review (ICR) of $47.5 \%$ in the Phase 2 (NCT02760498) primary analysis of the weight-based dosing cohort for patients with mCSCC (Group 1) with emerging evidence of durable responses. ${ }^{18}$ Supported by these findings, cemiplimab-rwlc became the first therapy approved by the US Food and Drug Administration for the treatment of advanced CSCC. ${ }^{19}$ Subsequently, the European Commission granted conditional marketing authorization for cemiplimab for the treatment of advanced CSCC. ${ }^{20}$ The approved regimen is cemiplimab $350 \mathrm{mg}$ every 3 weeks (Q3W) intravenously.

This article presents the primary analysis of the Phase 2 study of the approved fixed dose regimen (cemiplimab $350 \mathrm{mg}$ intravenously Q3W; Group 3) in patients with mCSCC. At the time of the Group 3 primary analysis, an additional data cut with longer follow-up was performed in Group 1 (cemiplimab $3 \mathrm{mg} / \mathrm{kg}$ intravenously every 2 weeks $(\mathrm{Q} 2 \mathrm{~W}))$ and reported here; results of the primary analysis of Group 1 have been previously reported. ${ }^{18}$ Exploratory TMB analyzes are also presented.

\section{METHODS \\ Patients}

This is an open-label, non-randomized, multicenter, international, Phase 2 study of patients with distant or nodal mCSCC (Groups 1 and 3) (see online supplementary file 1, S1 for study sites and principal investigators). Enrollment for Group 3 opened after full enrollment of Group 1 . The time point for the primary analysis of data from patients in Group 3 was reached.

Eligible patients were aged $\geq 18$ years with histologically confirmed diagnosis of invasive CSCC, an Eastern Cooperative Oncology Group performance status score of 0 or 1 , adequate organ function, and at least one measurable lesion per Response Evaluation Criteria in Solid Tumors version 1.1 (RECIST 1.1). ${ }^{21}$

Key exclusion criteria included ongoing or recent (within 5 years) autoimmune disease requiring systemic immunosuppression, prior treatment with an agent blocking the PD-1/PD ligand-1 (PD-L1) pathway, a history of solid organ transplantation, concurrent cancer (unless indolent or non-life-threatening), or hematologic cancer.

\section{Study design}

Patients were administered cemiplimab $350 \mathrm{mg}$ intravenously over $30 \mathrm{~min} \mathrm{Q} 3 \mathrm{~W}$ for up to 54 weeks, with the option to extend treatment to 96 weeks (Group 3) or cemiplimab $3 \mathrm{mg} / \mathrm{kg}$ intravenously over $30 \mathrm{~min}$ Q2W for up to 96 weeks (Group 1). The primary endpoint was ORR per ICR independently in each group. Tumor assessments were performed at the end of each treatment cycle (every 9 weeks for Group 3 and every 8 weeks for Group 1) (see online supplementary file 1, S2 for further details).

Secondary endpoints included ORR per investigator review (INV), duration of response (DOR) by ICR and INV, progression-free survival (PFS) by ICR and INV, overall survival (OS), complete response (CR) rate per ICR, adverse events (AEs), and quality of life. Durable disease control rate, defined as the proportion of patients with response or stable disease for at least 105 days was also examined. Safety assessments included treatment-emergent AEs (TEAEs), laboratory tests, vital signs, and physical examinations. The severity of TEAEs was graded according to the National Cancer Institute Common Terminology Criteria for Adverse Events (version 4.03).

Archived tumor samples from prior CSCC biopsies or surgeries were provided during the screening period. TMB was estimated in DNA samples extracted from formalin-fixed paraffin-embedded tumor biopsies. TMB was calculated as the total number of somatic single nucleotide variants and indels in the coding regions of targeted genes per megabase of analyzed genomic sequence (see online supplmentary file 1, S2 for further details).

The study also includes Group 4 and pilot Group 5, which explore alternative doses and/or schedules of cemiplimab, and Group 6 which is designed to confirm the results with $350 \mathrm{mg}$ intravenously Q3W observed in Group 3. Groups 4 to 6 have not reached primary analysis and are not included in this report.

\section{Statistical analysis}

The primary endpoint analyses for each group were statistically independent. Fifty patients in each group were needed to provide at least $85 \%$ power to reject a null hypothesis of an ORR of $15 \%$ at a two-sided significance level of no more than $5 \%$, if the true ORR was $34 \%$. The primary efficacy analyses were undertaken using $95 \%$ binomial exact confidence intervals (CIs), which were generated using the Clopper-Pearson method. ${ }^{22}$ The secondary efficacy analyses of DOR, PFS, and OS are summarized by their medians and $95 \%$ CIs, which were generated by the Kaplan-Meier method. CR rates are summarized descriptively. The full analysis set, which included all patients who passed screening and were eligible for study participation, was used for the analysis of all efficacy endpoints. The safety analysis set included all enrolled patients who received any study drug. The primary efficacy analysis of both groups was performed 6 months after the first dose of cemiplimab had been administered to the last patient enrolled; the results from the primary efficacy analysis of Group 1 (cut-off date of October 27, 2017) have been previously published. ${ }^{18}$ In the present analysis, data are reported separately for Group 1 and Group 3, as well as for both groups combined. The cut-off date for the primary analysis of Group 3 and follow-up analysis of Group 1 was September 20, 2018. 
Table 1 Baseline demographics and disease characteristics

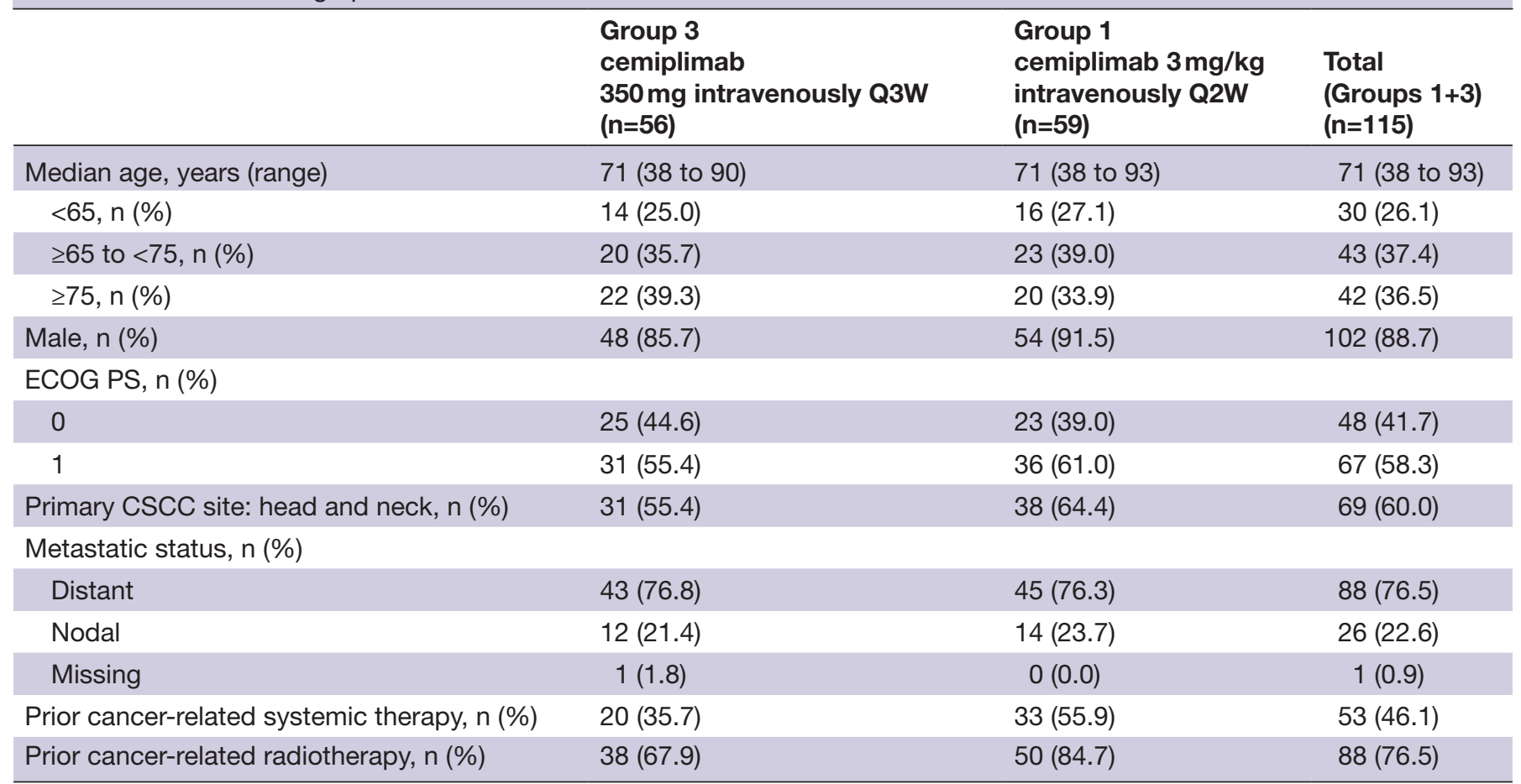

CSCC, cutaneous squamous cell carcinoma; ECOG PS, Eastern Cooperative Oncology Group performance status; Q2W, every 2 weeks; Q3W, every 3 weeks.

\section{RESULTS}

\section{Patients}

Results are presented for Group 3 primary analysis, and for Group 1 with approximately 11 months additional follow-up after the primary analysis. In total, 56 patients were enrolled and treated with cemiplimab $350 \mathrm{mg}$ Q3W (Group 3) from July 2017 through March 2018, and 59 patients were enrolled and treated with cemiplimab $3 \mathrm{mg} / \mathrm{kg}$ Q2W (Group 1) from March 2016 through January 2017. Baseline characteristics were similar in regard to median age, gender, and extent of disease (nodal vs distant) (table 1). The median duration of follow-up was 8.1 (range, 0.6 to 14.1) months for Group 3 and 16.5 (range, 1.1 to 26.6) months for Group 1. Patients in Group 3 received a median of 11.5 (range, 1 to 20) doses of cemiplimab and were exposed to treatment for a median of 34.3 (range, 2.6 to 60.4) weeks. Patients in Group 1 received a median of 31.0 (range, 1 to 48) doses of cemiplimab and were exposed to treatment for a median of 65.0 (range, 2.0 to 96.1) weeks. Patient disposition is summarized in online supplementary file 2 , figure S1.

\section{Clinical activity}

For the Group 3 primary analysis, ORR per ICR was $41.1 \%$ $(95 \%$ CI, $28.1 \%$ to $55.0 \%)$. For the Group 1 update, ORR per ICR was $49.2 \%$ (95\% CI, $35.9 \%$ to $62.5 \%)$. The combined ORR for both groups was $45.2 \%$ (95\% CI, $35.9 \%$ to $54.8 \%$ ), including 39 partial responses (PRs) and 13 CRs per ICR (table 2). The ORR per INV was $51.8 \%(95 \%$ CI, $38.0 \%$ to $65.3 \%)$ in Group 3 and $49.2 \%$
(95\% CI, $35.9 \%$ to $62.5 \%$ ) in Group 1 (see online supplementary file 3 , table $\mathrm{S} 1$ ).

Per ICR, most evaluable patients in both Group 3 and Group 1 had a decrease from baseline in the target lesion diameters (figure 1), and most responses were evident at the first response assessment in both groups (see online supplementary file 2, figure S2). Durable responses are most evident in Group 1 due to longer follow-up and are emerging for Group 3 (see online supplementary file 2, figure S2). The median DOR had not been reached in either group at data cut-off. Per ICR, the Kaplan-Meier estimate for DOR at 8 months was $95.0 \%$ (95\% CI, $69.5 \%$ to $99.3 \%$ ) in responding patients in Group 3, and at 12 months was $88.9 \%$ (95\% CI, $69.3 \%$ to $96.3 \%$ ) in responding patients in Group 1 (table 2).

The median time to response per ICR was 2.1 (range, 2.0 to 8.3 ) months for Group 3, 1.9 (range, 1.7 to 9.1) months for Group 1, and 2.1 (range, 1.7 to 9.1) months for both groups combined. The disease control rate per ICR was $64.3 \%$ (95\% CI, $50.4 \%$ to $76.6 \%$ ) in Group 3, $71.2 \%$ (95\% CI, $57.9 \%$ to $82.2 \%$ ) in Group 1, and $67.8 \%$ $(95 \%$ CI, $58.5 \%$ to $76.2 \%)$ in both groups combined. The durable disease control rate per ICR was $57.1 \%(95 \%$ CI, $43.2 \%$ to $70.3 \%)$ in Group 3, $61.0 \%$ (95\% CI, $47.4 \%$ to $73.5 \%$ ) in Group 1, and $59.1 \%$ (95\% CI, $49.6 \%$ to $68.2 \%$ ) in both groups combined (table 2 ).

Median PFS had not been reached at the time of data cut-off. The median Kaplan-Meier estimated PFS per ICR was 10.4 (95\% CI, 3.6 to not evaluable (NE)) months based on $44.6 \%$ event rate for Group 3, 18.4 (95\% CI, 
Table 2 Tumor response per independent central review

\begin{tabular}{|c|c|c|c|}
\hline & $\begin{array}{l}\text { Group } 3 \\
\text { cemiplimab } \\
350 \text { mg intravenously } \\
\text { Q3W ( } n=56)\end{array}$ & $\begin{array}{l}\text { Group } 1 \\
\text { cemiplimab } \\
3 \mathrm{mg} / \mathrm{kg} \text { intravenously } \\
\text { Q2W ( }=59)\end{array}$ & $\begin{array}{l}\text { Total } \\
\text { (Groups } 1+3) \\
(n=115)\end{array}$ \\
\hline ORR, \% (95\% Cl) & 41.1 (28.1 to 55.0$)$ & 49.2 (35.9 to 62.5$)$ & 45.2 (35.9 to 54.8$)$ \\
\hline \multicolumn{4}{|l|}{ Best overall response, n (\%) } \\
\hline Complete response & $3(5.4)$ & $10(16.9)$ & $13(11.3)$ \\
\hline Partial response & $20(35.7)$ & $19(32.2)$ & $39(33.9)$ \\
\hline Stable disease & $8(14.3)$ & $9(15.3)$ & $17(14.8)$ \\
\hline Non-complete response/non-progressive disease & $5(8.9)$ & $4(6.8)$ & $9(7.8)$ \\
\hline Progressive disease & $14(25.0)$ & $10(16.9)$ & 24 (20.9) \\
\hline Not evaluable & $6(10.7)$ & $7(11.9)$ & $13(11.3)$ \\
\hline Disease control rate, \% $(95 \% \mathrm{Cl})$ & 64.3 (50.4 to 76.6$)$ & 71.2 (57.9 to 82.2$)$ & 67.8 (58.5 to 76.2$)$ \\
\hline Durable disease control rate*, \% $(95 \% \mathrm{Cl})$ & 57.1 (43.2 to 70.3$)$ & $61.0(47.4$ to 73.5$)$ & 59.1 (49.6 to 68.2 ) \\
\hline Median time to response, months (range) ${ }^{\dagger}$ & 2.1 (2.0 to 8.3$)$ & $1.9(1.7$ to 9.1$)$ & $2.1(1.7$ to 9.1$)$ \\
\hline Median DOR & Not reached & Not reached & Not reached \\
\hline Kaplan-Meier 8-month estimate of DOR, \% $(95 \% \mathrm{Cl})^{\dagger}$ & 95.0 (69.5 to 99.3$)$ & 88.9 (69.3 to 96.3$)$ & 90.0 (75.2 to 96.2$)$ \\
\hline Kaplan-Meier 12-month estimate of DOR, \% $(95 \% \mathrm{Cl})^{\dagger}$ & Not evaluable & 88.9 (69.3 to 96.3$)$ & 90.0 (75.2 to 96.2$)$ \\
\hline
\end{tabular}

Errata: after database lock, the central review vendor noted an error in their initial assessment of one patient in Group 3 . This report contains the corrected data obtained after the vendor reviewed the case again. Also, the durable disease control rate for Group 1 was updated because one patient had tumor response after withdrawal of consent.

The ORR per investigator review was $51.8 \%(95 \% \mathrm{Cl}, 38.0 \%$ to $65.3 \%)$ in Group 3, $49.2 \%$ (95\% Cl, 35.9\% to $62.5 \%)$ in Group 1, and $50.4 \%$ $(95 \% \mathrm{Cl}, 41.0 \%$ to $59.9 \%)$ in both groups combined.

${ }^{*}$ Defined as the proportion of patients without progressive disease for at least 105 days.

†Data are based on number of patients with confirmed complete or partial response.

$\mathrm{Cl}$, confidence interval; DOR, duration of response; DOR, duration of response; ORR, objective response rate; ORR, objective response rate; Q2W, every 2 weeks; Q2W, every 2 weeks; Q3W, every 3 weeks; Q3W, every 3 weeks;

6.8 to NE) months based on $47.5 \%$ event rate for Group 1 , and 18.4 (95\% CI, 7.3 to NE) months based on $46.1 \%$ event rate for both groups combined. The Kaplan-Meier estimation of PFS at 12 months per ICR was $47.4 \%(95 \%$ CI, $29.6 \%$ to $63.3 \%$ ) for Group 3, $52.9 \%$ (95\% CI, $39.0 \%$ to $65.0 \%$ ) for Group 1, and $51.2 \%$ (95\% CI, $41.0 \%$ to $60.6 \%$ ) for both groups combined (see online supplementary file 2, figure S3A).

Median OS had not been reached in either group at data cut-off. The Kaplan-Meier estimation of OS at 12 months was $76.1 \%$ (95\% CI, $56.9 \%$ to $87.6 \%$ ) for Group 3, $81.3 \%$ (95\% CI, $68.7 \%$ to $89.2 \%$ ) for Group 1, and $80.7 \%$ (95\% CI, $71.9 \%$ to $87.1 \%$ ) for both groups combined (see online supplementary file 2, figure S3B).

For Group 3, ORR per ICR was explored for patients with high body weight $(>120 \mathrm{~kg})$. Among four patients who weighed $>120 \mathrm{~kg}$ at baseline, two experienced PR per ICR (see online supplementary file 3, table S2).

\section{Safety}

In both groups combined, 113 (98.3\%) patients experienced at least one TEAE of any grade regardless of attribution, including $96.4 \%$ for Group 3\% and $100.0 \%$ for Group 1 (table 3). The most common TEAEs in Group 3, Group 1, and both groups combined, were fatigue $(28.6 \%$,
$25.4 \%$, and $27.0 \%$, respectively), diarrhea (17.9\%, $28.8 \%$, and $23.5 \%$, respectively), and nausea $(17.9 \%, 23.7 \%$, and $20.9 \%$, respectively). Grade $\geq 3$ TEAEs regardless of attribution were reported in $45.2 \%$ of patients in both groups combined, with the most common in Group 3, Group 1, and both groups combined, being anemia $(8.9 \%, 3.4 \%$, and $6.1 \%$, respectively), fatigue $(5.4 \%, 1.7 \%$, and $3.5 \%$, respectively) and pneumonitis $(0.0 \%, 5.1 \%$, and $2.6 \%)$ (table 3). Treatment-related AEs (TRAEs) were reported in $71.3 \%(82 / 115)$ of patients, most commonly fatigue $(13.0 \%(15 / 115))$ (table 3; online supplementary file 3, table S2). TRAEs reported in $\geq 5 \%$ of patients in either treatment group are shown in online supplementary file 3, table S3. Immune-related AEs per INV are presented in online supplementary file 3 , table S4.

Three $(5.4 \%)$ patients in Group 3 discontinued treatment due to an $\mathrm{AE}$ (Grade 3 soft tissue necrosis, $\mathrm{n}=1$; Grade 2 lethargy, $\mathrm{n}=1$; and Grade 3 psoriasis, $\mathrm{n}=1$ ). In Group 1, six (10.2\%) patients discontinued treatment due to an AE; four of these were previously reported, ${ }^{18}$ and two occurred after the data cut for the Group 1 primary analysis. All AEs leading to treatment discontinuations were considered treatment-related, except for the patient in Group 3 with soft tissue necrosis on the 
A

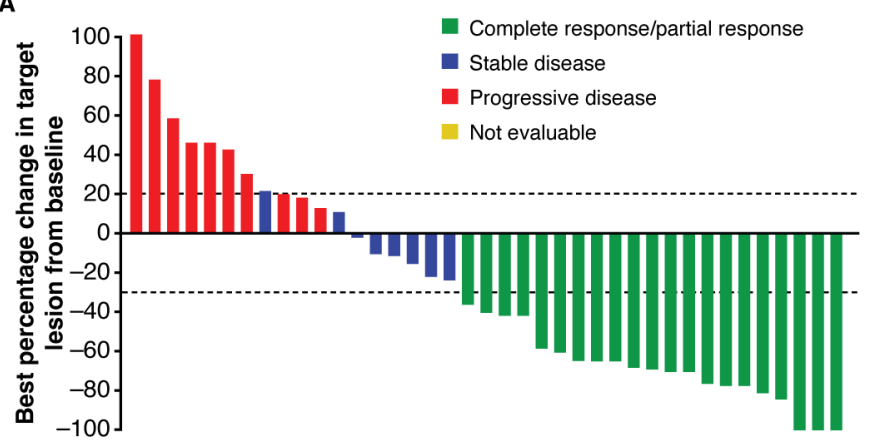

B

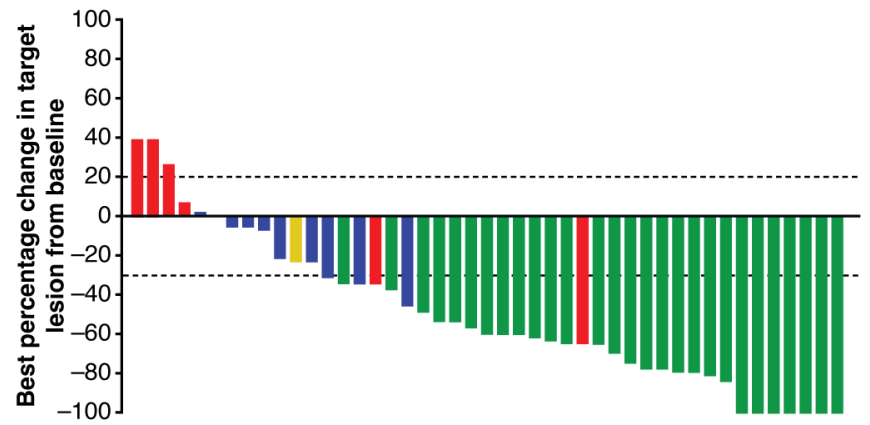

Figure 1 Best tumor response per RECIST 1.1 by independent central review for (a) Group 3 and (b) Group 1. This figure shows best percent change in the sum of tumor diameters for patients who had at least one postbaseline radiologic assessment (39 of 56 patients in Group 3 and 45 of 59 patients in Group 1). Lesion measurements after progression were excluded and patients who did not have at least one post-treatment radiologic assessment of target lesion(s) are not shown. The dashed lines indicate RECIST 1.1 criteria for partial response ( $\geq 30 \%$ decrease in sum of diameters) or progression ( $\geq 20 \%$ increase in sum of diameters) of target lesions. Patients with new lesions or unequivocal progression of non-target lesions are considered as progressive disease (red bars) regardless of target lesion response. Patients with a single assessment with $\geq 30 \%$ reduction of target lesion(s) are considered stable disease (blue bars) if there is not confirmatory assessment to establish partial response. One patient in Group 1 was not evaluable (NE) (yellow bar); this patient had radiologic and photographic data and was, therefore, reviewed by Independent Composite Review Committee and assessed as NE. Patients who did not have at least one evaluable postbaseline radiology assessment are not included in the figure but are included in the overall response analysis (table 2) per intention-to-treat. Increase in sum of target lesion diameters greater than $100 \%$ is reported as $100 \%$. RECIST 1.1 , Response Evaluation Criteria in Solid Tumors version 1.1.

head. One $(1.8 \%)$ patient in Group 3 died due to arterial hemorrhage from their right lower extremity tumor which measured $12.5 \mathrm{~cm}$ in longest diameter at baseline. This death was not considered related to study treatment. No new AEs resulting in death were reported in the updated analysis of Group 1.

\section{Biomarker analysis}

Overall, 79 patients had pre-treatment tumor samples available for the analysis of associations between TMB and clinical activity of cemiplimab. Median TMBs were 61.4 and 53.2 mutations per megabase among responding patients in Group 3 and Group 1 and were 13.7 and 19.4 mutations per megabase among non-responding patients in Group 3 and Group 1, respectively (see online supplementary file 2, figure S4). Similar separations in median TMB were observed among patients who achieved durable disease control and those who did not (see online supplementary file 2, figure S5).

\section{DISCUSSION}

The approved regimen of cemiplimab $350 \mathrm{mg}$ intravenously Q3W (Group 3) is highly active therapy for mCSCC (ORR per ICR, 41.1\%; 95\% CI $28.1 \%$ to $55.0 \%$ ). This result exceeds the prespecified statistical threshold for clinically meaningful ORR per ICR at time of primary analysis. The ORR per ICR for Group 1 at time of primary analysis was $47.5 \%,{ }^{18}$ and has increased to $49.2 \%$ with longer follow-up in this report. In the combined analysis of all mCSCC patients (Group 3 and Group 1) in this report, ORR per ICR is $45.2 \%$.

For the secondary endpoint of ORR per INV, numerical differences are smaller between the groups $(51.8 \%$ in Group 3, 49.2\% in Group 1). Discrepancies between ICR and INV are well described in oncology studies, ${ }^{23} 24$ and are more apparent in Group 3 than in Group 1 in this study. Despite these differences, the $95 \%$ CIs for ORR per ICR overlap broadly for the Group 3 primary analysis $(28.1 \%$ to $55.0 \%)$ and the Group 1 update $(35.9 \%$ to $62.5 \%)$. The characteristics of responses per ICR were similar for both groups in regard to the median time to response (2.1 and 1.9 months in Groups 3 and 1, respectively) and durability (estimated 8-month DOR of $95.0 \%$ and 12-month DOR of $88.9 \%$ in Groups 3 and 1, respectively). Numerical differences in point estimate of ORR per ICR between Group 3 and Group 1 are not attributed to differences between the two regimens, because the fixed-based and weight-based regimens display comparable pharmacokinetics, ${ }^{19}$ and exposure to cemiplimab in both groups was the same. ${ }^{25}$ The fixed-dose Group 3 regimen offers advantages such as a more convenient schedule for patients and less risk of dosing error or medication wastage. Cemiplimab $350 \mathrm{mg}$ Q3W intravenously is the commercially-approved dose. Additional clinical data regarding the fixed-dose regimen among patients with advanced CSCC are being obtained in a confirmatory cohort (Group 6) in this study.

The 11-month update of Group 1 illustrates hallmarks of the potential clinical benefits of PD-1 blockade that become apparent with longer follow-up. Almost all the Group 1 responses illustrated in the primary analysis report ${ }^{18}$ are still ongoing at this update (see online supplementary file 2, figure S2B). Additionally, the quality of responses improved over time, with 10 CRs per ICR at the 11-month update versus only four at the time of the primary analysis. ${ }^{18}$ Because the responses are sustained, the median DOR has yet to be reached. Therefore, the 
Table 3 Safety summary

\begin{tabular}{|c|c|c|c|c|c|c|}
\hline & \multicolumn{2}{|c|}{$\begin{array}{l}\text { Group } 3 \\
\text { cemiplimab } \\
350 \text { mg intravenously Q3W } \\
(n=56)\end{array}$} & \multicolumn{2}{|c|}{$\begin{array}{l}\text { Group } 1 \\
\text { cemiplimab } \\
3 \mathrm{mg} / \mathrm{kg} \text { intravenously Q2W } \\
(\mathrm{n}=59)\end{array}$} & \multicolumn{2}{|c|}{$\begin{array}{l}\text { Total } \\
\text { (Groups 1+3) } \\
(n=115)\end{array}$} \\
\hline & Any grade & Grade $\geq 3$ & Any grade & Grade $\geq 3$ & Any grade & Grade $\geq 3$ \\
\hline $\begin{array}{l}\text { Any TEAE, regardless of } \\
\text { attribution }\end{array}$ & $54(96.4)$ & $22(39.3)$ & $59(100.0)$ & $30(50.8)$ & $113(98.3)$ & $52(45.2)$ \\
\hline $\begin{array}{l}\text { TEAEs, regardless of attribution, } \\
\text { that led to discontinuation }\end{array}$ & $3(5.4)$ & $2(3.6)$ & $6(10.2)$ & $4(6.8)$ & $9(7.8)$ & $6(5.2)$ \\
\hline \multicolumn{7}{|c|}{ Most common TEAEs*, regardless of attribution } \\
\hline Fatigue & $16(28.6)$ & $3(5.4)$ & 15 (25.4) & $1(1.7)$ & $31(27.0)$ & $4(3.5)$ \\
\hline Diarrhea & $10(17.9)$ & $0(0.0)$ & $17(28.8)$ & $1(1.7)$ & 27 (23.5) & $1(0.9)$ \\
\hline Nausea & $10(17.9)$ & $0(0.0)$ & $14(23.7)$ & $0(0.0)$ & $24(20.9)$ & $0(0.0)$ \\
\hline Rash & $9(16.1)$ & $0(0.0)$ & $10(16.9)$ & $0(0.0)$ & $19(16.5)$ & $0(0.0)$ \\
\hline Constipation & $7(12.5)$ & $0(0.0)$ & $10(16.9)$ & $1(1.7)$ & $17(14.8)$ & $1(0.9)$ \\
\hline Pruritus & $6(10.7)$ & $0(0.0)$ & $10(16.9)$ & $0(0.0)$ & $16(13.9)$ & $0(0.0)$ \\
\hline Maculopapular rash & $7(12.5)$ & $1(1.8)$ & $8(13.6)$ & $0(0.0)$ & $15(13.0)$ & $1(0.9)$ \\
\hline Anemia & $7(12.5)$ & $5(8.9)$ & $7(11.9)$ & $2(3.4)$ & $14(12.2)$ & $7(6.1)$ \\
\hline Arthralgia & $5(8.9)$ & $0(0.0)$ & $9(15.3)$ & $0(0.0)$ & $14(12.2)$ & $0(0.0)$ \\
\hline Cough & $4(7.1)$ & $0(0.0)$ & $9(15.3)$ & $0(0.0)$ & $13(11.3)$ & $0(0.0)$ \\
\hline Headache & $2(3.6)$ & $0(0.0)$ & $11(18.6)$ & $0(0.0)$ & $13(11.3)$ & $0(0.0)$ \\
\hline Decreased appetite & $4(7.1)$ & $0(0.0)$ & $8(13.6)$ & $0(0.0)$ & $12(10.4)$ & $0(0.0)$ \\
\hline Hypothyroidism & $6(10.7)$ & $0(0.0)$ & $6(10.2)$ & $0(0.0)$ & $12(10.4)$ & $0(0.0)$ \\
\hline Vomiting & $6(10.7)$ & $0(0.0)$ & $6(10.2)$ & $0(0.0)$ & $12(10.4)$ & $0(0.0)$ \\
\hline Peripheral edema & $6(10.7)$ & $0(0.0)$ & $4(6.8)$ & $0(0.0)$ & $10(8.7)$ & $0(0.0)$ \\
\hline Upper respiratory tract infection & $3(5.4)$ & $0(0.0)$ & $6(10.2)$ & $0(0.0)$ & $9(7.8)$ & $0(0.0)$ \\
\hline Dizziness & $1(1.8)$ & $0(0.0)$ & $7(11.9)$ & $0(0.0)$ & $8(7.0)$ & $0(0.0)$ \\
\hline Dry skin & $2(3.6)$ & $0(0.0)$ & $6(10.2)$ & $0(0.0)$ & $8(7.0)$ & $0(0.0)$ \\
\hline Pneumonitis & $2(3.6)$ & $0(0.0)$ & $6(10.2)$ & $3(5.1)$ & $8(7.0)$ & $3(2.6)$ \\
\hline Dyspnea & $1(1.8)$ & $0(0.0)$ & $6(10.2)$ & $2(3.4)$ & $7(6.1)$ & $2(1.7)$ \\
\hline Oropharyngeal pain & $0(0.0)$ & $0(0.0)$ & $6(10.2)$ & $0(0.0)$ & $6(5.2)$ & $0(0.0)$ \\
\hline Treatment-related $^{\dagger}$ & $36(64.3)$ & $7(12.5)$ & $46(78.0)$ & $9(15.3)$ & $82(71.3)$ & $16(13.9)$ \\
\hline
\end{tabular}

Data are number of patients (\%).

${ }^{*}$ Adverse events reported in $\geq 10 \%$ of patients in either treatment group are presented, ordered by frequency in both groups combined. †See online supplement for additional details on treatment-related adverse events.

Q2W, every 2 weeks; Q3W, every 3 weeks; TEAEs, treatment-emergent adverse events.

protocol has been amended to allow another year of active follow-up with centrally reviewed imaging after completion of planned therapy. Patients in both groups continued in active follow-up after the data cut for this article, and long-term data continues to be collected so that the tail of the curve regarding survival and response duration can be more fully characterized in these groups.

Most TRAEs in both groups were Grades 1 to 2 and the discontinuation rate was low, regardless of attribution. The TEAE profile here is comparable to that of PD-1 checkpoint inhibitors pembrolizumab and nivolumab in patients with other cancer types such as head and neck squamous cell cancer. ${ }^{26}{ }^{27} \mathrm{~A}$ larger proportion of patients in the present analyses was aged at least 75 years $(36.5 \%$ vs $6.3 \%$ in pembrolizumab and $5.0 \%$ in nivolumab). ${ }^{26} 27$ The results presented here indicate that the safety profile with the anti-PD-1 class is not markedly different between younger and older patients. Overall, the safety profile for cemiplimab in this article continues to be consistent with that which has been reported for other anti-PD-1/PD-L1 inhibitors. $^{28}$

As data accumulate regarding the treatment of advanced CSCC patients with cemiplimab, the distinctions from results obtained in earlier studies of conventional therapies become clearer. This includes studies of cytotoxic chemotherapy and epidermal growth factor receptor-targeted therapy. ${ }^{67}$ Durable responses to these agents are not common, and the TEAEs associated 
with these therapies can be difficult to manage among older patients with advanced CSCC. Although there are currently no clinical trials directly comparing cemiplimab with these therapies, and the study design of the conventional therapies are different from that of cemiplimab, the differences in efficacy, durability of response, and safety position highlight cemiplimab as the standard of care for patients with advanced CSCC. In recent congress proceedings, pembrolizumab demonstrated ORR of $34.3 \%$ ( $n=105$; median follow-up of 9.5 months) in patients with recurrent/mCSCC. ${ }^{29}$ These data further support the clinical activity of PD-1 checkpoint inhibitors in advanced CSCC.

Higher median TMB was observed among responding patients than among non-responding patients in both Groups 3 and 1. Similar results were observed in exploratory analyses of laCSCC patients in Group $2 .{ }^{30}$ However, high TMB among some non-responders and low TMB among some responders preclude this assay from use as a patient selection tool. Future prospective data sets that integrate baseline TMB with other candidate biomarkers or clinical prognostic factors may better define features associated with clinical benefit among patients with advanced CSCC treated with cemiplimab.

In conclusion, cemiplimab $350 \mathrm{mg}$ intravenously Q3W produced substantial antitumor activity. Durable responses have been observed in both weight-based and fixed-dosing groups. The safety profile was similar in both groups. Long-term follow-up of these patients is ongoing.

\section{Author affiliations}

${ }^{1}$ Department of Medical Oncology, Peter MacCallum Cancer Centre, Melbourne, Victoria, Australia

${ }^{2}$ Departments of Dermatology and Head and Neck Surgery, University of Texas MD

Anderson Cancer Center, Houston, Texas, USA

${ }^{3}$ Department of Dermatology, Brigham and Women's Hospital, Harvard Medical School, Boston, Massachusetts, USA

${ }^{4}$ Department of Cutaneous Oncology, Moffitt Cancer Center, Tampa, Florida, USA

${ }^{5}$ Royal Brisbane and Women's Hospital and the University of Queensland, Brisbane, Queensland, Australia

${ }^{6}$ University Hospital Essen, Essen and German Cancer Consortium, Essen, Germany ${ }^{7}$ Department of Medicine, Head and Neck Medical Oncology, Memorial Sloan Kettering Cancer Center, New York, New York, USA

${ }^{8}$ Department of Medicine, Washington University School of Medicine, St Louis, Missouri, USA

${ }^{9}$ Department of Dermatology, Stanford University School of Medicine, Redwood City, California, USA

${ }^{10}$ Division of Dermatology, City of Hope, Duarte, California, USA

${ }^{11}$ Department of Dermatology, University Hospital (UKSH), Kiel, Germany

${ }^{12}$ Skin Cancer Centre, Charité Universitätsmedizin Berlin, Berlin, Germany

${ }^{13}$ Department of Dermatology, Center for Dermatooncology, University Medical

Center Tübingen, Tübingen, Germany

${ }^{14}$ Adelaide Cancer Centre, Adelaide, South Australia, Australia

${ }^{15}$ Department of Medical Oncology, New York University Langone Medical Center, New York, New York, USA

${ }^{16}$ Department of Hematology and Medical Oncology, Cleveland Clinic Taussig Cancer Institute, Cleveland, Ohio, USA

${ }^{17}$ Department of Dermatology and Allergy, Skin Cancer Center Hannover, Hannover

Medical School, Hannover, Germany

${ }^{18}$ Department of Dermatology, Northwestern University, Chicago, Illinois, USA

${ }^{19}$ Regeneron Pharmaceuticals, Inc, London, UK

${ }^{20}$ Regeneron Pharmaceuticals, Inc, Tarrytown, New York, USA

${ }^{21}$ Regeneron Pharmaceuticals, Inc, Basking Ridge, New Jersey, USA
${ }^{22}$ Department of Medical Oncology, Royal North Shore Hospital, St Leonards, New South Wales, Australia

Acknowledgements The authors would like to thank the patients, their families, all other study investigators, and all investigational site members involved in this study. Zhen Chen (Regeneron Pharmaceuticals, Inc) provided additional statistical analyses.

Contributors Responsibility for all opinions, conclusions, and data interpretation lies with the authors.

Funding This work was supported by Regeneron Pharmaceuticals, Inc. and Sanofi. Medical writing support, provided by Kate Carolan, PhD, of Prime (Knutsford, UK) according to Good Publication Practice guidelines (link), was funded by Regeneron Pharmaceuticals, Inc. and Sanofi.

Competing interests DR: institutional research grant and funding from Regeneron Pharmaceuticals, Inc., Sanofi, Roche, Merck Sharp \& Dohme, Bristol-Myers Squibb, and GlaxoSmithKline; uncompensated scientific committee and advisory board from Merck Sharp \& Dohme, Regeneron Pharmaceuticals, Inc., Sanofi, GlaxoSmithKline, and Bristol-Myers Squibb; travel and accommodation from Merck Sharp \& Dohme and GlaxoSmithKline. MRM: honoraria and travel expenses from Regeneron Pharmaceuticals, Inc., Sanofi, Novartis, Genentech, Eli Lilly, and Sun Pharma; and institutional research funding from Regeneron Pharmaceuticals, Inc., Novartis, Genentech, and Eli Lilly. AML: uncompensated advisory board from Merck Sharp \& Dohme and Bristol-Myers Squibb with travel and accommodation expenses. CDS: steering committee member for Castle Biosciences; a steering committee member and consultant for Regeneron Pharmaceuticals, Inc.; a consultant for Sanofi; has received research funding from Castle Biosciences, Regeneron Pharmaceuticals, Inc., Novartis, Genentech, and Merck, and is a chair for the National Comprehensive Cancer Network. NIK: grants from Regeneron Pharmaceuticals, Inc.; grants and advisory board fees from Bristol-Myers Squibb and HUYA Bioscience International; advisory board fees from EMD Serono, Regeneron Pharmaceuticals, Inc., Genentech, AstraZeneca (data safety monitoring committee), Merck, Array BioPharma, and Immunocore; grants from Merck, Novartis, GlaxoSmithKline, Celgene, and Amgen; honorarium from Sanofi; and common stock ownership of Bellicum Pharmaceuticals, Mazor Robotics, Amarin, and TransEnterix. BGMH: consulting or advisory roles at Merck Sharp \& Dohme, Bristol-Myers Squibb, AstraZeneca, Pfizer, Roche, Eisai, Merck, and institutional research funding from Amgen. DS: institutional patients' fees from Regeneron Pharmaceuticals, Inc.; advisory board honorarium fees from Amgen and Leo Pharma; speaker fee from Boehringer Ingelheim; advisory board, speaker honorarium and patients' fees from Roche, Novartis, Bristol-Myers Squibb, and Merck-EMD; advisory board and speaker honorarium fees from Incyte and Pierre Fabre; advisory board honorarium and patients' fees from Merck Sharp \& Dohme, steering committee honorarium fees from 4SC, advisory board fees from AstraZeneca, Pfizer, and Array; and advisory board and patients' fees from Philiogen. LAD: advisory role at Regeneron Pharmaceuticals, Inc., and research funding from Eisai, Pfizer, Regeneron Pharmaceuticals, Inc. LH-A: performed consulting and advisory roles at Massive Bio; speakers' bureau roles at Sanofi and Regeneron Pharmaceuticals, Inc., and received travel, accommodations, and expenses from Regeneron Pharmaceuticals, Inc., Sanofi, and Bristol-Myers Squibb, and research funding from Bristol-Myers Squibb, Regeneron Pharmaceuticals, Inc., Immunocore, Merck Sharp \& Dohme, Polynoma, Corvus Pharmaceuticals, Roche, Merck Serono, Amgen, Medlmmune, and Takeda. ALSC: consulting and advisory roles at Regeneron Pharmaceuticals, Inc., Merck; research funding from Regeneron Pharmaceuticals, Inc., Novartis, Galderma, and Merck. BM: speaker's honoraria, travel, accommodations and expenses from Regeneron Pharmaceuticals, Inc. and Sanofi. AH: institutional grants, speaker's honoraria, and consultancy fees from Amgen, Bristol-Myers Squibb, Merck Sharp \& Dohme/Merck, Pierre Fabre, Provectus, Roche, and Novartis; institutional grants and consultancy fees from Merck Serono, Philogen, and Regeneron Pharmaceuticals, Inc.; and consultancy fees from OncoSec. CU: honoraria, consulting, or advisory roles, speaker's bureau role, research funding and travel, accommodation, and expenses from Novartis, Sanofi, Galderma, and Almirall. TE: consulting or advisory roles at Sanofi Genzyme, Bristol-Myers Squibb, Roche, Novartis and Merck Sharp \& Dohme; speakers' bureau role at Roche and Merck Sharp \& Dohme and research funding from Novartis and Bristol-Myers Squibb. BS: consulting or advisory roles at Merck Sharp \& Dohme and Merck KGaA Australia. ACP: honoraria and consulting or advisory roles at Bristol-Myers Squibb, Merck, Regeneron Pharmaceuticals, Inc., Array, Novartis, Seattle Genetics, Amgen; research funding from Bristol-Myers Squibb, Merck, Regeneron Pharmaceuticals, Inc., Celldex, and Forance and travel, accommodation, expenses from Regeneron Pharmaceuticals, Inc., Array, and Seattle Genetics. JLG: research institution 
support for the study from and advisory board for Regeneron Pharmaceuticals, Inc. RG: honoraria from Almirall Hermal GmbH, Amgen, AstraZeneca, Bristol-Myers Squibb, Merck Serono, Merck Sharp \& Dohme, Novartis, Pierre Fabre, Regeneron Pharmaceuticals, Inc., Roche/Genentech, Sanofi, and Sun Pharma; consulting or advisory role for 4SC, Almirall Hermal GmbH, Amgen, Bristol-Myers Squibb, Incyte, LEO Pharma, Merck Serono, Merck Sharp \& Dohme, Novartis, Pierre Fabre, Roche/ Genentech, Sun Pharma, and Takeda; research funding from Amgen, Johnson \& Johnson, Novartis, and Pfizer; travel, accommodations, expenses from BristolMyers Squibb, Merck Sorono, Pierre Fabre, and Roche. MA: consulting or advisory roles for Pulse Biosciences and Revance Therapeutics. EO, MM, VJ, ES, JB, SL, IL, MGF: employees and shareholders of Regeneron Pharmaceuticals, Inc. AG: personal fees and non-financial support (advisory board and travel support) from BristolMyers Squibb and Sun Pharma; personal fees (advisory board) from Merck KGaA, Eisai, and Pfizer; non-financial (travel) support from Astellas; and clinical trial unit support from PPD Australia.

\section{Patient consent for publication Not required.}

Ethics approval This study was conducted in accordance with the principles of the Declaration of Helsinki and the International Conference on Harmonisation Good Clinical Practice guidelines. The study protocol and all amendments were approved by the institutional review board/ethics committee at each participating study site. All patients provided written informed consent.

Provenance and peer review Not commissioned; externally peer reviewed.

Data availability statement All data relevant to the study are included in the article or uploaded as supplementary information. Qualified researchers may request access to study documents (including the clinical study report, study protocol with any amendments, blank case report form, statistical analysis plan) that support the methods and findings reported in this manuscript. Individual anonymized participant data will be considered for sharing once the indication has been approved by a regulatory body, if there is legal authority to share the data and there is not a reasonable likelihood of participant re-identification. Submit requests to https://vivli.org/.

Open access This is an open access article distributed in accordance with the Creative Commons Attribution Non Commercial (CC BY-NC 4.0) license, which permits others to distribute, remix, adapt, build upon this work non-commercially, and license their derivative works on different terms, provided the original work is properly cited, appropriate credit is given, any changes made indicated, and the use is non-commercial. See http://creativecommons.org/licenses/by-nc/4.0/

\section{REFERENCES}

1 Lomas A, Leonardi-Bee J, Bath-Hextall F. A systematic review of worldwide incidence of nonmelanoma skin cancer. $\mathrm{Br} J$ Dermatol 2012;166:1069-80.

2 Rogers HW, Weinstock MA, Feldman SR, et al. Incidence estimate of nonmelanoma skin cancer (keratinocyte carcinomas) in the U.S. population, 2012. JAMA Dermatol 2015;151:1081-6.

3 Madan V, Lear JT, Szeimies R-M. Non-melanoma skin cancer. Lancet 2010;375:673-85.

4 Cranmer LD, Engelhardt C, Morgan SS. Treatment of unresectable and metastatic cutaneous squamous cell carcinoma. Oncologist 2010;15:1320-8.

5 Kim JYS, Kozlow JH, Mittal B, et al. Guidelines of care for the management of cutaneous squamous cell carcinoma. J Am Acad Dermatol 2018;78:560-78.

6 Stratigos A, Garbe C, Lebbe C, et al. Diagnosis and treatment of invasive squamous cell carcinoma of the skin: European consensusbased interdisciplinary guideline. Eur J Cancer 2015;51:1989-2007.

7 NCCN. National comprehensive cancer network clinical practice guidelines in oncology: squamous cell skin cancer (version 2.2019), 2018.

8 Karia PS, Jambusaria-Pahlajani A, Harrington DP, et al. Evaluation of American Joint Committee on Cancer, International Union Against Cancer, and Brigham and Women's Hospital tumor staging for cutaneous squamous cell carcinoma. J Clin Oncol 2014;32:327-34.
9 Weinberg AS, Ogle CA, Shim EK. Metastatic cutaneous squamous cell carcinoma: an update. Dermatol Surg 2007;33:885-99.

10 Schmults CD, Karia PS, Carter JB, et al. Factors predictive of recurrence and death from cutaneous squamous cell carcinoma: a 10-year, single-institution cohort study. JAMA Dermatol 2013;149:541-7.

11 Pickering CR, Zhou JH, Lee JJ, et al. Mutational landscape of aggressive cutaneous squamous cell carcinoma. Clin Cancer Res 2014;20:6582-92.

12 Chalmers ZR, Connelly CF, Fabrizio D, et al. Analysis of 100,000 human cancer genomes reveals the landscape of tumor mutational burden. Genome Med 2017:9:34.

13 Büttner R, Longshore JW, López-Ríos F, et al. Implementing TMB measurement in clinical practice: considerations on assay requirements. ESMO Open 2019;4:e000442.

14 Samstein RM, Lee C-H, Shoushtari AN, et al. Tumor mutational load predicts survival after immunotherapy across multiple cancer types. Nat Genet 2019;51:202-6.

15 Euvrard S, Kanitakis J, Claudy A. Skin cancers after organ transplantation. N Engl J Med 2003;348:1681-91.

16 Nehal KS, Bichakjian CK. Update on keratinocyte carcinomas. N Engl J Med 2018;379:363-74.

17 Burova E, Hermann A, Waite J, et al. Characterization of the antiPD-1 antibody REGN2810 and its antitumor activity in human PD-1 knock-in mice. Mol Cancer Ther 2017;16:861-70.

18 Migden MR, Rischin D, Schmults CD, et al. PD-1 blockade with cemiplimab in advanced cutaneous squamous-cell carcinoma. N Engl J Med 2018;379:341-51.

19 Regeneron Pharmaceuticals, Inc.. LIBTAYO $B$ [cemiplimabrwlc] injection full US prescribing information, 2018. Available: https://www.accessdata.fda.gov/drugsatfda_docs/label/2018/ 761097s000lbl.pdf [Accessed 23 Dec 2019].

20 European Medicines Agency. LIBTAYO ${ }^{8}$ EPAR, 2019. Available: https://www.ema.europa.eu/en/medicines/human/EPAR/libtayo [Accessed 23 Dec 2019].

21 Eisenhauer EA, Therasse P, Bogaerts J, et al. New response evaluation criteria in solid tumours: revised RECIST guideline (version 1.1). Eur J Cancer 2009;45:228-47.

22 Clopper CJ, Pearson ES. The use of confidence or fiducial limits illustrated in the case of the binomial. Biometrika 1934;26:404-13.

23 Zhang J, Zhang Y, Tang S, et al. Systematic bias between blinded independent central review and local assessment: literature review and analyses of 76 phase III randomised controlled trials in 45688 patients with advanced solid tumour. BMJ Open 2018;8:e017240.

24 Zhang J, Zhang Y, Tang S, et al. Evaluation bias in objective response rate and disease control rate between blinded independent central review and local assessment: a study-level pooled analysis of phase III randomized control trials in the past seven years. Ann Transl Med 2017:5:481.

25 Migden MR, Paccaly A, Papadopoulos KP, et al. 1279P Pharmacokinetic (PK) analysis of weight-based and fixed dose cemiplimab in patients (pts) with advanced malignancies. Ann Oncol 2019;30:v520.

26 Ferris RL, Blumenschein G, Fayette J, et al. Nivolumab for recurrent squamous-cell carcinoma of the head and neck. N Engl J Med 2016;375:1856-67.

27 Cohen EEW, Soulières D, Le Tourneau C, et al. Pembrolizumab versus methotrexate, docetaxel, or cetuximab for recurrent or metastatic head-and-neck squamous cell carcinoma (KEYNOTE-040): a randomised, open-label, phase 3 study. Lancet 2019;393:156-67.

28 Wang Y, Zhou S, Yang F, et al. Treatment-related adverse events of PD-1 and PD-L1 inhibitors in clinical trials: a systematic review and meta-analysis. JAMA Oncol 2019;5:1008-19.

29 Grob JJ, Gonzalez Mendoza R, Basset-Seguin N, et al. LBA72 Pembrolizumab for recurrent/metastatic cutaneous squamous cell carcinoma (cSCC): efficacy and safety results from the phase II KEYNOTE-629 study. Ann Oncol 2019;30:mdz394.069:v908.

30 Migden MR, Khushalani NI, Chang ALS, et al. Cemiplimab in locally advanced cutaneous squamous cell carcinoma: results from an open-label, phase 2, single-arm trial. Lancet Oncol 2020;21:294-305. 